\title{
Monte Carlo model of a polychromatic laboratory based edge illumination $x$-ray phase contrast system
}

\author{
T. P. Millard, ${ }^{1, \text { a) }}$ M. Endrizzi, ${ }^{1}$ P. C. Diemoz, ${ }^{1}$ C. K. Hagen, ${ }^{1}$ and A. Olivo ${ }^{1,}$ b) \\ Department of Medical Physics and Bioengineering, University College London, Malet Place, Gower Street, \\ London WC1E 6BT, UK
}

(Dated: 11 March 2014)

A Monte Carlo model of a polychromatic laboratory based (coded aperture) edge illumination x-ray phase contrast imaging system has been developed and validated against experimental data. The ability for the simulation framework to be used to model two-dimensional images is also shown. The Monte Carlo model has been developed using the McXtrace engine and is polychromatic, i.e. results are obtained through the use of the full x-ray spectrum rather than an effective energy. This type of simulation can in future be used to model imaging of objects with complex geometry, for system prototyping, as well as providing a first step towards the development of a simulation for modelling dose delivery as a part of translating the imaging technique for use in clinical environments.

\section{INTRODUCTION}

X-rays are used for imaging in a wide range of applications encompassing the clinical through to the industrial, with images normally formed based on x-ray absorption within the sample. Over recent years study in x-ray imaging has instead been focused on exploiting phase effects induced by the object, as for materials and energies commonly used this effect is much larger than absorption. For all applications this promises increased image contrast, whilst also revealing previously undetectable details due to the different physical principle behind contrast generation. For clinical uses, lower patient doses are also a possibility as a lower x-ray flux or higher x-ray energy should be required for equivalent contrast. ${ }^{1}$

Various methods have been developed to detect these object induced phase shifts, though the early methods were limited to spatially coherent x-ray sources. This made these first methods impractical as micro-focal sources currently provide limited x-ray flux leading to long exposure times. ${ }^{2-11}$ Greater flux is achieved at synchrotron facilities, but the scale of these makes them unsuitable as an x-ray source for the widespread adoption of x-ray phase contrast imaging (XPCi).

To enable using XPCi in the same settings as absorption based imaging a method was required that works with an x-ray tube. The grating based Talbot-Lau interferometer made a step towards this, as the introduction of a source grating allowed for such a source to be used. $^{12,13}$ A clinical system based on this method has been developed to image articular cartilage in the hand, with this XPCi system revealing details invisible in a conventional absorption image. ${ }^{14}$ In addition to this recent developments have also shown progress in implementing analyser crystal based XPCi methods with conventional X-ray tubes. ${ }^{15-18}$

\footnotetext{
a) Electronic mail: t.millard@ucl.ac.uk

b) Electronic mail: a.olivo@ucl.ac.uk
}

This work looks instead at the coded aperture edge illumination XPCi method (here referred to just as edge illumination XPCi), which uses masks with a much larger pitch and works with an x-ray tube. ${ }^{19,20}$ This noninterferometric method has recently been shown to have comparable phase sensitivity to grating interferometry. ${ }^{21}$ Figure 1 shows a diagram of an edge illumination XPCi system where the beam is split into an array of minibeams which, when deviated by a sample, create contrast through movement either on to or off of the detector mask apertures.

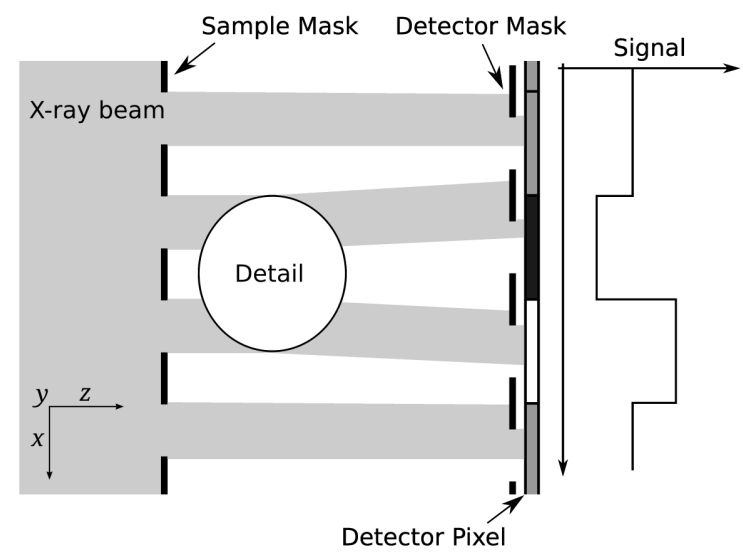

FIG. 1: A simplified diagram of an edge illumination $\mathrm{x}$-ray phase contrast system, depicting how a detail imaged with the system generates contrast.

A single image taken with the system will contain a mixture of absorption and phase signals, and by taking images with the sample mask illuminating either side of the detector apertures these can be separated. ${ }^{22}$ In addition, higher resolution images can be taken by shifting the sample by sub-pixel steps and then interleaving these to create a 'dithered' image.

Simulation of this type of system has already been carried out using geometrical optics ${ }^{23}$ and the FresnelKirchhoff theory of diffraction ${ }^{24,25}$, with previous study 
showing that for an extended focal spot geometrical optics and Fresnel-Kirchoff theory produce equivalent results. ${ }^{24,26}$ A simple voxelized geometrical optics based model has also been shown for an edge illumination system. ${ }^{27}$ This work was not though quantitative, due to the particular voxelized approach used as well as the use of opaque mask strips and monochromatic x-ray beam.

Getting a direct correspondence between a polychromatic simulation and experimental image is challenging as the response of all components in the system to $\mathrm{x}$ ray energy has to be understood. An effective energy is instead usually chosen so that a monochromatic simulation can be used, which is though problematic as it has been shown that effective energy depends on the imaged object. $^{28}$

A Monte Carlo model can be made inherently polychromatic through sampling of an x-ray spectrum and using look up tables to determine $\mathrm{x}$-ray refractive index. Moreover, a Monte Carlo model provides no limits to the orientation of components in the imaging system as the often used paraxial approximation is relaxed. All of this means that the development of a Monte Carlo model of the edge illumination XPCi system would allow for system prototyping that is not possible with current Fresnel-Kirchhoff based models.

The field of view it is possible to simulate with current Fresnel-Kirchhoff based models is limited as the entire wavefront needs to be finely sampled and held in memory. A model such as that proposed here has no such limitation as each ray is simulated individually. The computational power available with current desktop computers allows for the simulation of fields of view orders of magnitude larger than that possible with Fresnel-Kirchhoff based models. Propagation of each ray can be computed in parallel, with the code used here already suitable for use with supercomputing facilities.

Here a fully polychromatic Monte Carlo model of an edge illumination XPCi system is set out and validated through simulation of wires using parameters matching those used experimentally. Wires were chosen for simulation work as they provide a clear and unambiguous refraction and absorption signal with a geometry that is easily defined.

The model presented in this work builds on the previously presented synchrotron results showing a match between simulation and experiment for ultra-small angle scatter signal from microbubbles imaged using analyser based imaging. ${ }^{29}$ The same microbubble code used in this previous work can be included in the edge illumination simulation framework presented here. This will in the future allow for the simulation of the ultra-small angle scatter signal from microbubbles using edge illumination.

\section{MODEL}

The model presented here is based on the McXtrace Monte Carlo engine, ${ }^{30}$ which is optimised for parallel computing architectures via Message Parsing Interface (MPI), though the results set out are generic to other Monte Carlo frameworks. In essence the system geometry is defined in a file termed the 'instrument' file, and components within the system are set out in files termed 'component' files. Through writing additional $\mathrm{C}$ code component files, and a custom instrument file, an arbitrary x-ray imaging system can be modelled. More detail on the structure and function of McXtrace can be found in Knudsen et al. 2013. ${ }^{30}$

To allow for McXtrace to be used to model an edge illumination system, appropriate masks and sample had to be coded. Furthermore, data are required to describe all source parameters including the source spectrum, material refractive indices, and geometry.

The output spectrum of the x-ray source was calculated using the data tables provided by Boone et al. ${ }^{31}$ which consist of polynomials fitted to the measured spectral output of molybdenum target x-ray tubes. Spectra calculated from this model were then corrected for the differing filtration before being used as input to the simulation, with the focal spot in the simulation modelled using a three-dimensional Gaussian determining the probability of event emission.

For this work xraylib ${ }^{32}$ was used as the source of the refractive index data, with data tables created from this. In principle McXtrace could directly call xraylib to access this data, but this would need to be incorporated into McXtrace at source code level. Direct access to xraylib would then remove the need to interpolate from lookup tables.

Current experimental masks consist of a $500 \mu \mathrm{m}$ thick graphite substrate with electroplated strips of gold forming the absorbing parts. All x-rays passing through the mask will be attenuated by the graphite substrate. Whether the specific photon passes through the gold strip or not is then calculated using the function $T$ expressed in Eq. 1, where $p$ is the mask period, $x$ is the location of the event on the mask $x$-axis and $w$ is the mask aperture width. Attenuation is found according to the energy of the incident ray and the complex refractive index values from the graphite and, when needed, gold data tables.

$$
T(x, y)=\left\{\begin{array}{l}
1 \text { if } \cos \left(\frac{2 \pi x}{p}\right) \geq \cos \left(\frac{\pi w}{p}\right) \\
0, \text { otherwise }
\end{array}\right.
$$

Refraction caused by the wire phantom implemented here is calculated through a vector form of Snell's law, and the Beer-Lambert law is used to calculate attenuation. When an event reaches the wire, a function is called to determine whether the event intersects the wire, and if this is the case the intersection point is stored in memory. A surface normal is calculated and the refractive index for that event's energy is retrieved from the appropriate data table. After the event's direction has been modified according to refraction the event is propagated again to find if or where it exits the wire, and refraction is calculated at the exit point. Using the entrance 
and exit points, the distance travelled through the wire is found, and attenuation taken into account by adjusting the event's weight. The event is then propagated forward in the simulation.

All of the experimental results shown here are the result of multiple exposures acquired at sub-pixel sample displacement, which are then recombined to form high resolution dithered images, which means that multiple simulations are also required. This was all controlled through MATLAB, which was used to set commonly varied system parameters, launch simulations, read in and combine dithered images, perform normalisation and plot data. The result of this is a user-friendly interface to perform Monte Carlo simulations of an edge illumination XPCi system, that can be used also with limited knowledge on the specific technical details of the imaging method.

\section{SYSTEM}

All experimental data shown here were acquired with the same prototype edge illumination XPCi system, with figure 2 showing an image of the sample mask, detector mask and detector arrangement used.

An Anrad SMAM detector, with $85 \mu \mathrm{m}$ pixels, is mounted on an optical bench with masks mounted on drive units to allow for automated alignment and imaging. ${ }^{33}$ A Rigaku MicroMax 007 HF x-ray source with molybdenum target was placed $2 \mathrm{~m}$ from the detector with masks, made to the authors' design by Creatv MicroTech Inc. (Potomac, MD, USA), placed at approximately 1.57 and $1.96 \mathrm{~m}$ from the source respectively. The sample is mounted directly behind the sample mask on a third drive unit, which allows for dithering to be performed to a high degree of accuracy. A pre-collimator is used directly after the x-ray source that defines the beam shape so it almost exactly matches the sample mask.

This system uses a sample mask with a pitch of 66.8 $\mu \mathrm{m}$ and apertures of $12 \mu \mathrm{m}$, and a detector mask with pitch of $83.5 \mu \mathrm{m}$ and apertures of $20 \mu \mathrm{m}$. The sample mask is $48 \times 48 \mathrm{~mm}$ and the detector mask is $60 \times 60 \mathrm{~mm}$ in size.

\section{ATTENUATION CURVE}

Before any polychromatic simulations of the edge illumination XPCi system can be performed, one first needs to find whether the simulation can model the sourcedetector combination without the masks present. If this simplest case is not achieved, then further simulations will not be possible either; furthermore any errors at this stage are likely to propagate through and impact on edge illumination simulations.

An attenuation curve can be measured by progressively adding known thicknesses of a given material without

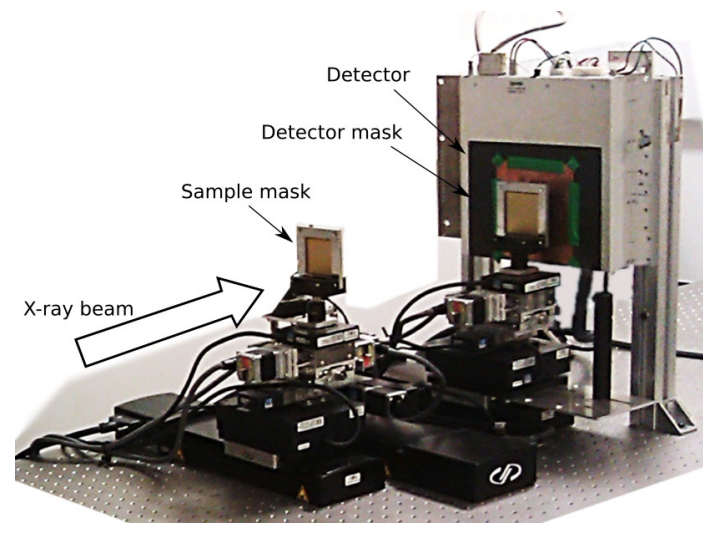

FIG. 2: Image of the prototype laboratory based edge illumination system.

masks present and measuring the change in detected signal. To accurately model this, the source spectrum and detector energy response have to be known. The Anrad SMAM detector is a direct conversion amorphous selenium detector, and to a first approximation its energy response can be assumed to be directly proportional to x-ray energy. Simulations performed using this approximation give an attenuation curve for which transmission decreases slightly more rapidly than seen experimentally (black dashed line figure 3). This suggests that the relative importance of high x-ray energies is underestimated in the model.

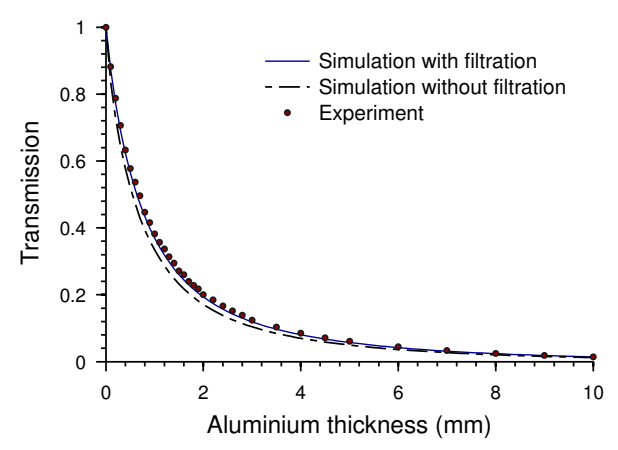

FIG. 3: Attenuation curve for aluminium taken at 40 $\mathrm{kVp}$ with the molybdenum x-ray source (red dots). The simulation was performed using the approximation to the detector response (black dashed line) and an additional $1.5 \mathrm{~mm}$ carbon filter (blue solid line).

The materials protecting the detector components are unknown, but a carbon fibre cover can clearly be seen on the front of the detector. By including (before the detector) a $1.5 \mathrm{~mm}$ layer of material of the same complex refractive index as used to model the mask graphite, the result shown in figure 3 (blue solid line) is achieved. This additional carbon filter in front of the active detector components is reasonable, and provides a good basis for moving towards a full simulation of the edge illumina- 
tion XPCi system. Future work will look into fully characterising this detector, and once performed this will be incorporated into further simulation work.

\section{ILLUMINATION CURVES}

By scanning the sample mask in the $x$ direction (figure 1) a curve is mapped out which is related to the system's imaging performance. ${ }^{21}$ Thus, before the imaging of an object can be simulated, a match is needed to these curves. As can be seen in both figure 4a and figure $4 \mathrm{~b}$, when the masks are positioned with the apertures completely mismatching, x-rays are still detected at both 35 and $40 \mathrm{kVp}$. This is due to transmission through the gold parts of the masks, which is energy dependent. As a consequence, for a polychromatic model, inputting an accurate gold thickness is fundamental to obtain a correspondence between simulation and experiment.

As discussed previously, in an ideal case mask strips would be fully absorbing. With a polychromatic spectrum, especially with future designs aimed at operating at higher energies, this may not prove possible, which highlights the importance of performing simulation work that takes mask transmission into account.

This was included in the simulations by introducing two masks into the McXtrace instrument file used to model the attenuation curve, both of which were given the pitch and aperture size of that used experimentally. Both masks were placed parallel to the detector, in the $z$ position resulting in the projected pitches matching that of the detector pixels, with apertures oriented vertically. Detector mask apertures were then positioned so that they aligned with the centre of the detector pixels, and the sample mask was set so that its $x$ position could be controlled from the MATLAB interface. All geometry described above is the same as used experimentally. Illumination curves are then created in both simulation and experiment by stepping the sample mask in sub-pitch steps, finding the mean of an image at each step, and plotting the result.

System illumination curves were measured by stepping the sample mask in $2 \mu \mathrm{m}$ steps over an entire period. Mask thickness and source size within the simulation were then found through iterative adjustment until a match was found between simulation and experiment, with $28.3 \mu \mathrm{m}$ thick gold and a $67 \mu \mathrm{m}$ FWHM source size giving a reasonably good fit to the illumination curves at the energies used in this work (figure 4). It is important to note that the only difference between simulation results shown in figures $4 \mathrm{a}$ and $4 \mathrm{~b}$ is the input spectrum, which was also the only parameter that was changed experimentally.

The mask thickness and source size found are in reasonable agreement with specifications and previous observations. Previous source sizes reported have been in the region of $70 \mu \mathrm{m}$ FWHM, and gold thickness was specified to the manufacturer as $30 \mu \mathrm{m}$. Even so these two
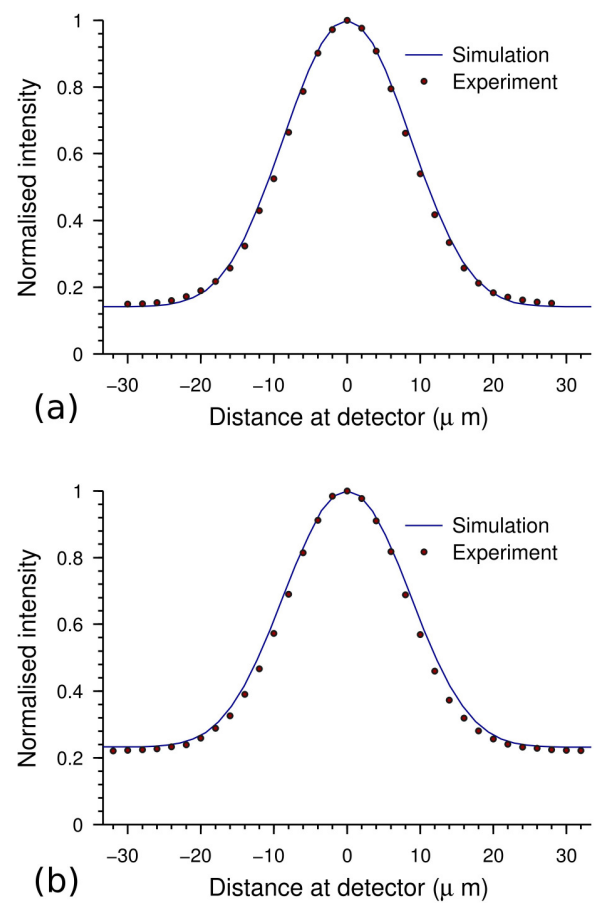

FIG. 4: Illumination curves, and corresponding simulation, showing results for the spectra and system parameters used for all simulations. (a) Illumination curve taken at $35 \mathrm{kVp}$. (b) Illumination curve taken at $40 \mathrm{kVp}$.

values should not be seen as a definitive measurement, but instead the real value slightly distorted by unavoidable errors propagated from the source spectrum, detector response, and potentially even attenuation coefficient data used.

\section{PROFILES}

Simulating a wire with the Monte Carlo model simply requires adding the wire function to the instrument file, providing it with geometry parameters, setting the sample mask to the correct position, and selecting the correct source spectrum. All experimental results presented here use 8 dithering steps, with the aim of finely sampling the refraction peaks in the profiles. Simulations were then performed using 32 dithering steps so that in the simulation peak maximum and minimum were definitely not missed.

All simulations were performed using $7.7 \times 10^{6}$ events per pixel per dithering step, which took approximately 6 seconds per step running on a standard desktop computer with an Intel Core i7 2600s processor. This gives the potential for performing even more complex simulations, as a single simulation run is quick.

PEEK is a material that provides a strong refraction signal but little absorption at the considered energies, 


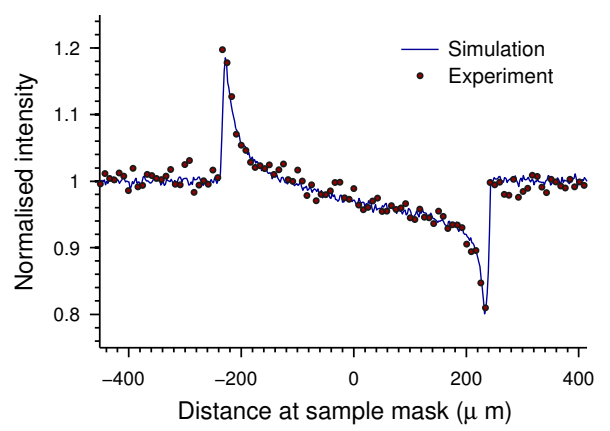

FIG. 5: Profile of a PEEK wire, diameter $482 \mu \mathrm{m}$, imaged at $40 \mathrm{kVp}$. The simulation was performed using the same system parameters as for the illumination curve in figure $4 \mathrm{~b}$, apart from the fixed sample mask position and addition of the wire.

making it a good first material to test. An experimental and simulated profile of a $482 \mu \mathrm{m}$ diameter PEEK wire, imaged at $40 \mathrm{kVp}$, is shown in figure 5 , with the sample mask in the simulation translated along the $x$ axis by 9.3 $\mu \mathrm{m}$ from aperture alignment to match the experiment.

The fit between simulation and experiment for this low absorbing object appears good. However, in a real use case absorption will also be present. To test the simulation of an absorbing object, a sapphire wire with diameter of $250 \mu \mathrm{m}$, and a $200 \mu \mathrm{m}$ diameter boron wire with $14 \mu \mathrm{m}$ diameter tungsten core were imaged at $35 \mathrm{kVp}$ with a $+8 \mu \mathrm{m}$ sample mask offset; the results of this are shown in figure 6 . The tungsten core was simulated by placing a tungsten wire directly behind the boron wire, which is a reasonable approximation thanks to boron's low absorption. Future developments will though allow for objects to be directly embedded within each other in the simulation.

For both the sapphire wire (figure $6 \mathrm{a}$ ) and boron wire (figure 6b) a good match is again seen for the refraction peaks. This shows the flexibility of the simulation approach as through changing diameter, refractive index data, and spectrum a good fit is seen to this different experimental data.

There is though some discrepancy due to absorption through the thickest part of the wire, which can be seen for the sapphire wire profile (figure 6a) . It is not though possible to tell from the tungsten data the degree of difference as the minimum of the profile has not been sampled in the experiment because of the very small diameter. This difference is due to a difference in the detection of lower energy x-rays in the simulation, which probably relates back to the imperfectly known detector response. Solving this problem requires precise measurements of source spectrum and detector response.

The simulation was then tested against experimental data of two PEEK wires imaged using the sample mask shifted in the opposite direction by $-8 \mu \mathrm{m}$, which causes the maximum and minimum peaks to flip. The exper-
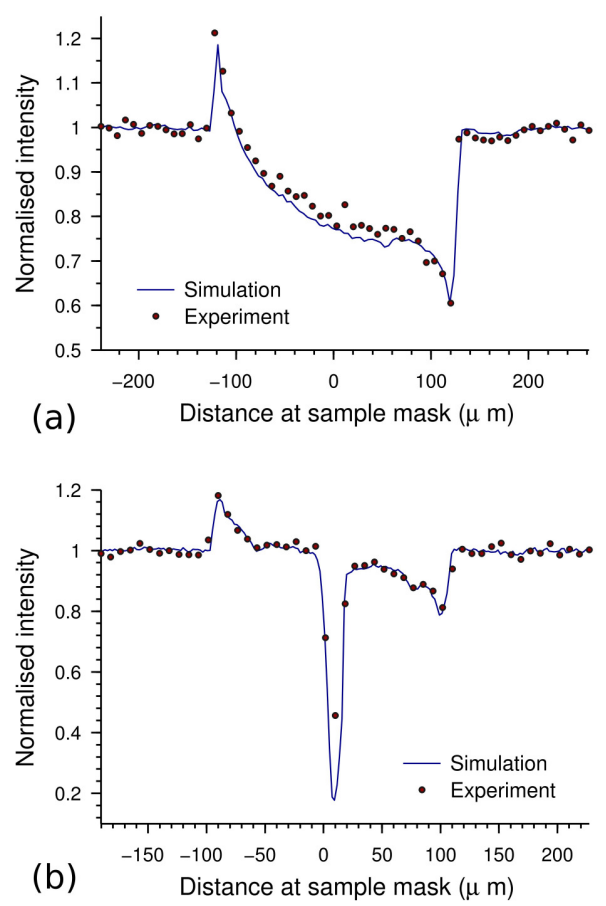

FIG. 6: (a) Profile of a sapphire (diameter $250 \mu \mathrm{m}$ ) and (b) boron wire with tungsten core (diameter 200 and 14 $\mu \mathrm{m}$ respectively), imaged at $35 \mathrm{kVp}$.

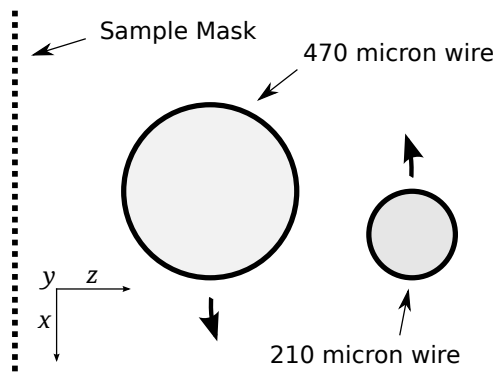

FIG. 7: Diagram showing how the wires were positioned for the experimental and simulation results shown in figure 8 . The two wires were attached to a rotation stage, rotation of which allowed for change in the amount of overlap seen in a projection image.

imental data was acquired at $40 \mathrm{kVp}$ with the PEEK wires $(470$ and $210 \mu \mathrm{m})$, placed at $50 \mathrm{~mm}$ and $120 \mathrm{~mm}$ from the sample mask respectively. The arrangement of the two wires is shown in figure 7. By attaching the wires vertically to a mount on a rotation stage it was possible to adjust the position of the wires to change the overlap seen in a projection image. In figure 8a the two wires are positioned so that they are directly behind each other, and in figure $8 \mathrm{~b}$ the wires have been rotated so that the maximum peaks overlap.

A monochromatic McXtrace model, using an effective energy, has previously been compared against the experimental data shown in figure $8 .{ }^{34}$ These previous results 
used masks with opaque strips, and included no model for the detector response. Results shown in figure 8 demonstrate that the edge illumination signal can be modelled just as well without having to use an effective energy. Instead by modelling the full source spectra, and mask structure, the same match is achieved with the experimental data.
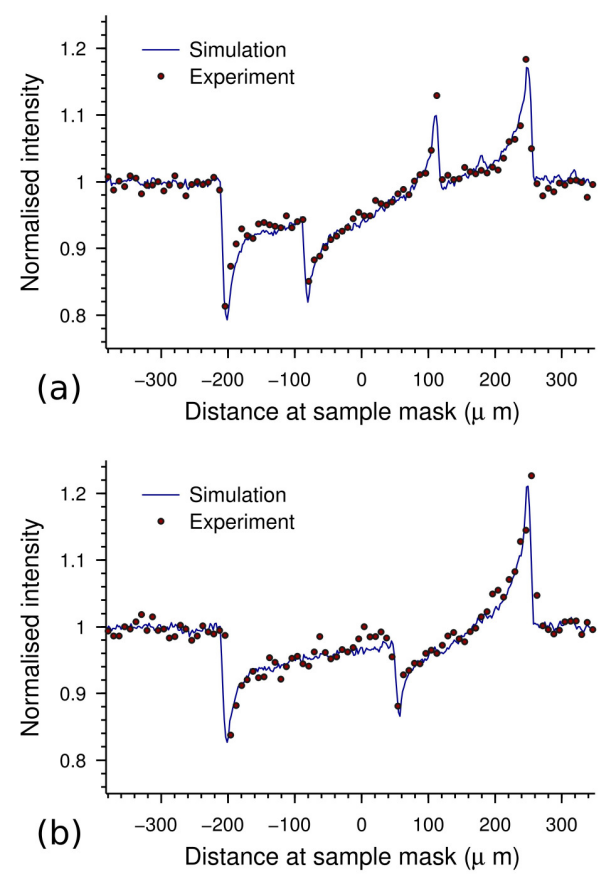

FIG. 8: Experimental profiles, and corresponding simulations, of two PEEK wires (diameter 470 and 210 $\mu \mathrm{m}$ ) imaged at $40 \mathrm{kVp}$. (a) Both wires positioned with centre at $x=0$. (b) Wires positioned so the maximum peaks overlap.

After changing sample mask position, spectrum, and wire thickness in simulation and experiment, as good a fit is seen for the two wires in figure 8 as figure 5 . The combined effect of two wires also produces an equivalent result in experiment and simulation, providing evidence of simulation accuracy even when the complexity of the imaged sample is slightly increased. This demonstrates the simulation's amenability to 'modular' approaches, thus showing promise for the simulation of more complex scenarios.

\section{IMAGES}

An advantage of the Monte Carlo framework used is that it is inherently three dimensional, which means that no code change is required for extension to creating images instead of profiles. To generate an image the instrument file is simply adjusted to specify a two dimensional detector. As all code is written to be orientation invariant this means that masks, wires and detector can all be rotated in three dimensions with the imaging sequence performed as before. This can be done just through modification of parameters in the instrument file.

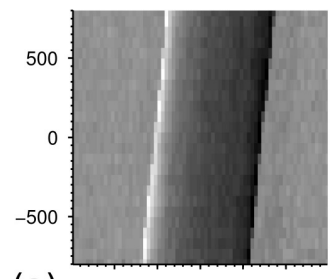

(a) $-200-100 \quad 0 \quad 100200$

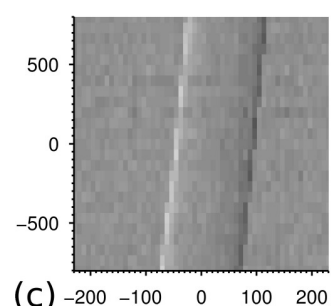

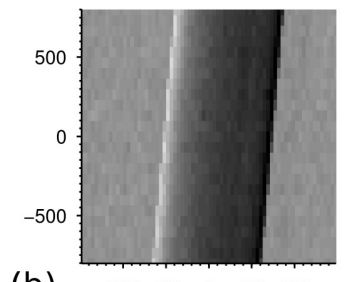

(b) $\quad-200-100 \quad 0 \quad 100200$

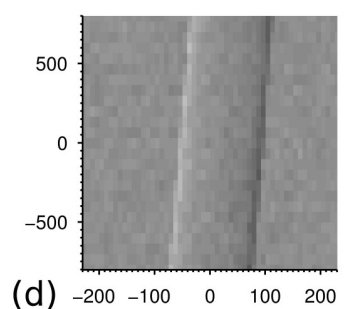

FIG. 9: Experimental image of a sapphire (a) and

PEEK wire (c), with diameters 250 and $150 \mu \mathrm{m}$

respectively, at $35 \mathrm{kVp}$. (b) Simulated image of the sapphire wire. (d) Simulated image of the PEEK wire. All images are displayed on the same scale with axes showing distance in micrometers at the sample mask.

Figure 9 shows the result of experiment and simulation of a sapphire and PEEK wire; these images are made up of 8 dithering steps each with a 7 second exposure to achieve a good balance between image statistics, sampling and total exposure time. The angle of rotation of the sapphire and PEEK wire about the $z$ axis was calculated from the experimental images, and found to be $1.86^{\circ}$ and $1.30^{\circ}$ respectively, which was then input to the simulation. The number of events used in the simulation was then calculated by matching the standard deviation of a background region in experiment and simulation, sample mask was positioned to $+8 \mu \mathrm{m}$ and a $35 \mathrm{kVp}$ spectrum was used. The resulting simulations show a good visual agreement, clearly demonstrating the different appearance of the sapphire and PEEK wires in the system.

Careful study of the wire edges in these images reveals that border intensity fluctuates, which is due to inadequate sampling of the refraction peaks and could be overcome by using more dithering steps. These results though show that this under sampling effect can be correctly reproduced using the Monte Carlo model.

Being able to freely rotate and move system components in three dimensions opens up the possibility of fully prototyping systems through simulation. This includes for example testing the tolerance of systems to mask misalignment through simulation of imaging with various degrees of mask rotation or misplacement, prototyping masks giving two-dimensional phase sensitivity ${ }^{35,36}$, or modelling three dimensional imaging in computed to- 
mography. The fully polychromatic nature of the simulation also allows for mask plating thickness, source spectra, and detectors to be prototyped, as well as testing alternative materials other than gold for mask plating.

A further use of a Monte Carlo model for such a system is to model dose throughout the system. This is of particular importance with the edge illumination method, where a mask before the sample splits the x-ray beam. During a single exposure, radiation dose over the sample is uneven, as thin blades of radiation pass through the sample. A dithered acquisition results in the eventual irradiation of the whole sample, but in certain applications it could easily be imagined that a dithered image would not be required. As well as this there is also the possibility of designing imaging sequences which exploit the principle of using a thin radiation beam to actually minimise dose delivered to the whole sample. All of this requires Monte Carlo modelling, and this work provides the basis for moving towards the development of a model taking into account the physical interactions required to model dose deposition.

\section{CONCLUSION}

A Monte Carlo model has been introduced that can be used to model a coded aperture edge illumination XPCi system. This model uses the best available information for the system to model polychromatic signal without using approximations such as effective energy. Such an approach will be used for testing future system designs as it allows for signal to be modelled using a polychromatic source, is inherently three dimensional allowing for testing of more complicated mask designs, as well as making it possible to test a system's tolerance to misalignment. In the future, Monte Carlo modelling will also be important for modelling dose delivery as a part of the method's translation to clinical environments; this work is a first step towards this ultimate goal.

\section{ACKNOWLEDGEMENTS}

This work was supported by the UK Engineering and Physical Sciences Research Council (Grant Nos. EP/G004250/1 and EP/I021884/1). M.E. and P.C.D. are supported by Marie Curie Career Integration Grant Nos. PCIG12-GA-2012-334056 and PCIG12-GA-2012333990 within the Seventh Framework Programme of the European Union.

${ }^{1}$ A. Bravin, P. Coan, P. Suortti, X-ray phase-contrast imaging: from pre-clinical applications towards clinics, Phys. Med. Biol. 58 (2013) R1.

${ }^{2}$ V. E. Cosslett, W. C. Nixon, The x-ray shadow microscope, J. Appl. Phys. 24 (1953) 616-623.

${ }^{3}$ U. Bonse, M. Hart, An x-ray interferometer, Appl. Phys. Lett. 6 (1965) 155-156.
${ }^{4}$ E. Förster, K. Goetz, P. Zaumseil, Double crystal diffractometry for the characterization of targets for laser fusion experiments, Krist. Tech. 15 (1980) 937-945.

${ }^{5}$ K. Podurets, V. Somenkov, S. S. Shil'shteTn, Refraction-contrast radiography, Sov. Phys. Tech. Phys. 34 (1989) 654-657.

${ }^{6}$ T. J. Davis, D. Gao, T. E. Gureyev, A. W. Stevenson, S. W. Wilkins, Phase-contrast imaging of weakly absorbing materials using hard x-rays, Nature 373 (1995) 595-598.

${ }^{7}$ A. Snigirev, I. Snigireva, V. Kohn, S. Kuznetsov, I. Schelokov, On the possibilities of $\mathrm{x}$-ray phase contrast microimaging by coherent high-energy synchrotron radiation, Rev. Sci. Instrum. 66 (1995) $5486-5492$.

${ }^{8}$ A. Momose, T. Takeda, Y. Itai, K. Hirano, Phase contrast x-ray computed tomography for observing biological soft tissues, Nat. Med. 2 (1996) 473-475.

${ }^{9}$ S. W. Wilkins, T. E. Gureyev, D. Gao, A. Pogany, A. W. Stevenson, Phase-contrast imaging using polychromatic hard x-rays, Nature 384 (1996) 335-338.

${ }^{10}$ C. David, B. Nohammer, H. H. Solak, E. Ziegler, Differential xray phase contrast imaging using a shearing interferometer, Appl. Phys. Lett. 81 (2002) 3287-3289.

${ }^{11} \mathrm{~A}$. Momose, Phase-sensitive imaging and phase tomography using x-ray interferometers, Opt. Express 11 (2003) 2303-2314.

${ }^{12}$ F. Pfeiffer, T. Weitkamp, O. Bunk, C. David, Phase retrieval and differential phase-contrast imaging with low-brilliance x-ray sources, Nat. Phys. 2 (2006) 258-261.

${ }^{13}$ F. Pfeiffer, J. Herzen, M. Willner, M. Chabior, S. Auweter, M. Reiser, F. Bamberg, Grating-based x-ray phase contrast for biomedical imaging applications, Z. Med. Phys. 23 (2013) 176185.

${ }^{14}$ A. Momose, W. Yashiro, K. Kido, J. Kiyohara, C. Makifuchi, T. Ito, S. Nagatsuka, C. Honda, D. Noda, T. Hattori, T. Endo, M. Nagashima, J. Tanaka, X-ray phase imaging: from synchrotron to hospital, Phil. Trans. R. Soc. A. 372 (2014) 20130023.

${ }^{15}$ D. Chapman, I. Nesch, M. Hasnah, T. Morrison, X-ray optics for emission line x-ray source diffraction enhanced systems, Nucl. Instrum. Meth. A. 562 (2006) $461-467$.

${ }^{16}$ C. Parham, Z. Zhong, D. M. Connor, L. D. Chapman, E. D. Pisano, Design and implementation of a compact low-dose diffraction enhanced medical imaging system, Acad. Radiol. 16 (2009) 911-917.

${ }^{17}$ B. Bewer, D. Chapman, Development of an x-ray prism for analyzer based imaging systems, Rev. Sci. Instrum. 81 (2010) 085108.

${ }^{18}$ M. G. Hönnicke, J. Manica, I. Mazzaro, C. Cusatis, X.-R. Huang, Source effects in analyzer-based x-ray phase contrast imaging with conventional sources, Rev. Sci. Instrum. 83 (2012) 113702.

${ }^{19}$ A. Olivo, F. Arfelli, G. Cantatore, R. Longo, R. Menk, S. Pani, M. Prest, P. Poropat, L. Rigon, G. Tromba, An innovative digital imaging set-up allowing a low-dose approach to phase contrast applications in the medical field, Med. Phys. 28 (2001) 16101619.

${ }^{20}$ A. Olivo, R. Speller, A coded-aperture technique allowing x-ray phase contrast imaging with conventional sources, Appl. Phys. Lett. 91 (2007) 074106.

${ }^{21}$ P. C. Diemoz, C. K. Hagen, M. Endrizzi, A. Olivo, Sensitivity of laboratory based implementations of edge illumination x-ray phase-contrast imaging, Applied Physics Letters 103 (2013) -.

${ }^{22}$ P. R. Munro, K. Ignatyev, R. D. Speller, A. Olivo, Phase and absorption retrieval using incoherent x-ray sources, Proc. Natl. Acad. Sci. U.S.A. 109 (2012) 13922-13927.

${ }^{23}$ A. Olivo, R. Speller, Modelling of a novel x-ray phase contrast imaging technique based on coded apertures., Phys. Med. Biol. 52 (2007) 6555-6573.

${ }^{24}$ P. R. T. Munro, K. Ignatyev, R. D. Speller, A. Olivo, The relationship between wave and geometrical optics models of coded aperture type x-ray phase contrast imaging systems, Opt. Express 18 (2010) 4103-4117.

${ }^{25}$ F. A. Vittoria, P. C. Diemoz, M. Endrizzi, L. Rigon, F. C. Lopez, D. Dreossi, P. R. T. Munro, A. Olivo, Strategies for efficient and 
fast wave optics simulation of coded-aperture and other x-ray phase-contrast imaging methods, Appl. Opt. 52 (2013) 69406947.

${ }^{26}$ A. Peterzol, A. Olivo, L. Rigon, S. Pani, D. Dreossi, The effects of the imaging system on the validity limits of the ray-optical approach to phase contrast imaging, Med. Phys. 32 (2005) 36173627.

${ }^{27}$ A. Kavanagh, A. Olivo, R. Speller, B. Vojnovic, Feasibility testing of a pre-clinical coded aperture phase contrast imaging configuration using a simple fast monte carlo simulator, Biomed. Opt. Express 5 (2014) 93-105.

${ }^{28}$ P. R. T. Munro, A. Olivo, X-ray phase-contrast imaging with polychromatic sources and the concept of effective energy, Phys. Rev. A 87 (2013) 053838.

${ }^{29}$ T. P. Millard, M. Endrizzi, L. Rigon, F. Arfelli, R. H. Menk, J. Owen, E. Stride, A. Olivo, Quantification of microbubble concentration through x-ray phase contrast imaging, Appl. Phys. Lett. 103 (2013) 114105.

${ }^{30}$ E. Bergbäck Knudsen, A. Prodi, J. Baltser, M. Thomsen, P. Kjær Willendrup, M. Sanchez del Rio, C. Ferrero, E. Farhi, K. Haldrup, A. Vickery, R. Feidenhans'l, K. Mortensen, M. Meedom Nielsen, H. Friis Poulsen, S. Schmidt, K. Lefmann, $M c X-$ trace: a Monte Carlo software package for simulating X-ray optics, beamlines and experiments, J. Appl. Crystallogr. 46 (2013)
679-696.

${ }^{31}$ J. M. Boone, T. R. Fewell, R. J. Jennings, Molybdenum, rhodium, and tungsten anode spectral models using interpolating polynomials with application to mammography, Med. Phys. 24 (1997) 1863-1874.

${ }^{32}$ T. Schoonjans, A. Brunetti, B. Golosio, M. S. del Rio, V. A. Sol, C. Ferrero, L. Vincze, The xraylib library for x-raymatter interactions. recent developments, Spectrochim. Acta. B. 66 (2011) $776-784$.

${ }^{33}$ T. P. Millard, M. Endrizzi, K. Ignatyev, C. K. Hagen, P. R. T. Munro, R. D. Speller, A. Olivo, Method for automatization of the alignment of a laboratory based x-ray phase contrast edge illumination system, Rev. Sci. Instrum. 84 (2013) 083702.

${ }^{34}$ M. B. Szafraniec, T. P. Millard, K. Ignatyev, R. D. Speller, A. Olivo, Proof-of-concept demonstration of edge-illumination $\mathrm{x}$ ray phase contrast imaging combined with tomosynthesis, Phys. Med. Biol. 59 (2014) N1.

${ }^{35}$ A. Olivo, S. E. Bohndiek, J. A. Griffiths, A. Konstantinidis, R. D. Speller, A non-free-space propagation x-ray phase contrast imaging method sensitive to phase effects in two directions simultaneously, Appl. Phys. Lett. 94 (2009) 044108.

${ }^{36}$ P. R. T. Munro, K. Ignatyev, R. D. Speller, A. Olivo, Design of a novel phase contrast x-ray imaging system for mammography, Phys. Med. Biol. 55 (2010) 4169. 